\title{
RELAÇÕES ENTRE EXPERIÊNCIA DE LEITURA, HABILIDADES LINGUÍSTICAS E ACESSO LEXICAL NA L2
}

\author{
ASSOCIATING READING EXPERIENCE, LANGUAGE SKILLS AND LEXICAL ACCESS \\ IN THE L2
}

\begin{abstract}
Pietra Cassol Rigatti ${ }^{19}$
Ana Beatriz Arêas da Luz Fontes ${ }^{20}$

RESUMO: A literatura aponta a importância da experiência de leitura para o desenvolvimento de uma L1 e o aprendizado de uma L2, em especial ao se tratar do acesso lexical em uma L2 (PERFETTI; HART, 2001; ARTIEDA, 2007). Além disso, as habilidades de leitura e de escrita são aprimoradas, podendo influenciar uma à outra. O objetivo deste estudo foi investigar as relações entre a experiência de leitura em L2, as habilidades autoavaliadas de leitura e de escrita em L2 e o acesso lexical em L2. Para tanto, 82 estudantes universitários bilíngues português-inglês responderam um questionário de histórico de uso de língua e um de hábitos de leitura em L2 e realizaram uma tarefa de decisão de significado na sua L2, em que decidiam se uma palavra-alvo era relacionada semanticamente a uma palavra-prime. Os resultados indicaram correlações negativas fracas entre as medidas de acesso lexical na L2 e as habilidades de leitura e escrita na L2; correlações positivas fracas entre a experiência de leitura em L2 e as habilidades de leitura e escrita na L2; e correlação positiva e moderada entre as habilidades de leitura e de escrita. Não houve correlação significativa entre o acesso lexical na L2 e a experiência de leitura na L2, o que foi inesperado. Esses achados corroboram a Hipótese da Qualidade Lexical, a Hipótese do Atraso de Frequência e estudos que relacionam os processos de leitura e escrita. A ausência da correlação entre acesso lexical na L2 e experiência de leitura na L2 difere da literatura e é discutida relativamente a falhas metodológicas.
\end{abstract}

Palavras-chave: Experiência de leitura; habilidades linguísticas; acesso lexical; bilinguismo.

\begin{abstract}
Literature indicates the importance of reading experience to L1 development and L2 learning, especially considering L2 lexical access (PERFETTI; HART, 2001; ARTIEDA, 2007). Moreover, reading and writing skills are improved with reading, and they may influence one another. The objective of this study was to investigate the associations with L2 reading experience, self-reported L2 reading and writing skills, and L2 lexical access. Thus 82 Portuguese-English university students filled a language use history questionnaire and a L2 reading habits questionnaire, and completed an L2 meaning decision task in which they decided whether a target word was semantically related to a prime word or not. Results presented negative weak correlations between L2 lexical access measures and L2 reading and writing skills; positive weak correlations between L2 reading experience and L2 reading and writing skills; and a positive moderate correlation between the L2 reading and writing skills themselves. No significant correlation was found between L2 lexical access and L2 reading experience, which was unexpected. These findings corroborate the Lexical Quality Hypotheses and the Frequency-Lag Hypotheses and also studies connecting reading and writing processes. The absence of a correlation between L2 lexical access and L2 reading experience differs from the literature and is discussed based on methodological flaws.
\end{abstract}

Keywords: Reading experience; language skills; lexical access; bilingualism.

\section{Introdução}

Uma atividade frequentemente recomendada para o aprendizado ou aprimoramento do conhecimento de uma segunda língua (L2) - ou até mesmo de uma primeira língua (L1) - é a leitura. De fato, essa ideia já foi e ainda é estudada, e existem evidências de que essa recomendação realmente funciona. Por exemplo, investigou-se a importância da ampliação de conhecimentos específicos sobre as palavras para uma leitura eficiente e também o quanto a experiência de leitura

\footnotetext{
${ }^{19}$ Doutoranda em Linguística pela Universidade Federal de Santa Catarina-UFSC. Membro do grupo de pesquisa Laboratório da Linguagem e Processos Cognitivos (LabLing) na UFSC.

${ }^{20}$ Doutora em Psicologia Social, Cognitiva e Neurociência pela The University of Texas at El Paso. Professora do Programa de Pós-Graduação em Letras da Universidade Federal do Rio Grande do Sul-UFRGS.
} 
e a exposição a materiais escritos podem contribuir para esses processos (PERFETTI; HART, 2001, 2002; TAYLOR; PERFETTI, 2016).

Assim, hábitos de leitura podem proporcionar o desenvolvimento de habilidades linguísticas relacionadas, como habilidades de leitura e de escrita. Parece haver certa bidirecionalidade no relacionamento entre leitura e escrita (PARODI, 2007; LEE; SCHALLERT, 2016; WONG, 2018). Ambas influenciam a automatização dos processos mais básicos de leitura, o que leva a um reconhecimento de palavras mais eficiente e que libera recursos cognitivos para processos mais exigentes, como a compreensão de leitura e a geração de inferências (PERFETTI; HART, 2001). Nesse sentido, o acesso lexical, especialmente em uma L2, tem muito a ser beneficiado das experiências de leitura do leitor.

Este estudo pretende investigar as relações entre a experiência de leitura em L2, representada pelos hábitos de leitura em L2, as habilidades autoavaliadas de leitura e de escrita na L2 e o acesso lexical na L2 de estudantes universitários bilíngues que têm o português brasileiro como L1 e o inglês como L2.

\section{Referencial teórico}

\subsection{Experiência de leitura}

A experiência de leitura é um conceito que faz parte da Hipótese da Qualidade Lexical (HQL) desenvolvida por Perfetti e Hart (2001, 2002). De acordo com Perfetti (2007, p. 3), experiência de leitura é a "prática efetiva" do conhecimento sobre constituintes de palavras. Ela é um dos fatores que afetam a qualidade lexical, que é o conhecimento sobre os constituintes ortográfico, fonológico, morfossintático e semântico das palavras. A experiência de leitura afeta a qualidade lexical pois, quanto mais lemos e mais estamos expostos a textos, mais conhecimento teremos sobre os aspectos lexicais e mais especificados estarão os constituintes das palavras. É a experiência de leitura que oferece aos leitores conhecimento lexical útil para aumentar a qualidade das representações lexicais (PERFETTI; HART, 2002).

A qualidade lexical depende da frequência das palavras. Uma palavra de alta frequência para um leitor que lê frequentemente será acessada facilmente devido aos diversos encontros que esse leitor teve com essa palavra, os quais contribuíram para especificar os constituintes lexicais. Entretanto, uma palavra de baixa frequência para um leitor ocasional causará confusão e irá interromper a leitura. Perfetti e Hart (2001) observaram esses efeitos de frequência em pares de palavras homófonas, que compartilham pronúncia, mas têm significados e grafia diferentes. Leitores habilidosos demonstraram confusão apenas ao lerem o membro de baixa frequência do par de palavras homófonas, enquanto leitores menos habilidosos foram confundidos por ambos os membros. Tais confusões com a forma de palavras, seja fonológica ou ortográfica, como no caso de homófonos e homógrafos, são causadas pela frequência de ocorrência dessas palavras (PERFETTI, 2007). Por exemplo, gate (portão) e gait (andar) são pronunciadas da mesma forma; porém, a segunda palavra ocorre com menos frequência que a primeira e pode causar confusão para um leitor pouco experiente. Esses efeitos de frequência têm impacto no processamento de palavras e na desambiguação (PERFETTI; HART, 2001). Além da frequência de ocorrência de palavras, a familiaridade ou frequência relativa deve ser considerada; ela é a experiência de um leitor com determinada palavra. Entretanto, essa experiência varia entre pessoas e "depende da quantidade de leitura feita" (PERFETTI; HART, 2001, p. 7). Desse modo, a experiência de leitura contribui para a estabilidade dos constituintes das palavras: mais leitura leva a mais encontros com palavras, o que promove o aumento da qualidade lexical desse leitor.

Os efeitos da experiência de leitura e, consequentemente, de constituintes lexicais de alta qualidade podem ser vistos durante o processamento de palavras que carregam algum tipo de ambiguidade, como homófonas, homógrafas, homônimas e polissêmicas. Nesses casos, uma 
representação ortográfica ou fonológica não pode ser mapeada diretamente a uma única representação semântica. Para leitores com menor experiência de leitura, será preciso mais tempo (TAYLOR; PERFETTI, 2016) ou mais recursos cognitivos (HAMILTON; FREED; LONG, 2016) para resolver essas ambiguidades do que para leitores mais experientes.

Ao mesmo tempo em que a experiência de leitura reforça a qualidade das representações lexicais, ela também parece estar relacionada ao tamanho do vocabulário. Pratheeba e Krashen (2013) investigaram a relação entre a experiência de leitura e o vocabulário em uma segunda língua. Para isso, um questionário de 20 perguntas sobre hábitos de leitura em L2 foi construído e respondido por participantes com variadas L1 e inglês como L2. O questionário incluía perguntas sobre leitura de materiais impressos e digitais, e suas opções de respostas seguiam uma escala de 5 pontos, de 0 , equivalendo a "nunca", a 5, "sempre". As respostas do questionário foram analisadas em relação às de um teste de vocabulário e, de modo geral, apresentaram uma correlação positiva significativa. O subconjunto de perguntas sobre a leitura de livros mostrou uma correlação moderada com o teste de vocabulário enquanto apenas uma das perguntas do subconjunto sobre a leitura digital - leitura por prazer - estava positivamente correlacionada com o vocabulário. Esses resultados indicam que a experiência de leitura é importante para desenvolver a qualidade lexical, porque pontuações maiores no teste de vocabulário estavam relacionadas a uma maior frequência de leitura. Assim, um vocabulário maior promove mais atividades de leitura, as quais alimentam esse mesmo léxico (PRATHEEBA; KRASHEN, 2013).

Esse impacto da experiência de leitura ocorre tanto em uma L1 quanto em uma L2. Por exemplo, Santos-Díaz (2017) demonstrou que a frequência de leitura de livros está relacionada positivamente com o tamanho de vocabulário em segundas línguas. O vocabulário específico em inglês aumentou conforme a leitura nessa língua se tornou mais frequente. $\mathrm{O}$ mesmo não pode ser dito para a primeira língua, espanhol. De forma geral, a autora concluiu que a leitura em L2 contribui para o desenvolvimento do vocabulário na L2.

O estudo de Hamada e Koda (2008) sugere que tanto a exposição a materiais escritos em L1 quanto as propriedades ortográficas do insumo da L2 podem impactar as habilidades de decodificação na L2. Esse resultado pode ser comparado com a conclusão de Pratheeba e Krashen (2013, p. 1) de que "a maior exposição a materiais escritos resulta em mais leitura, e mais leitura resulta no desenvolvimento da alfabetização, incluindo vocabulário”. Também é possível fazer um paralelo com a afirmação de que a experiência de leitura incrementa a qualidade lexical e de que o conhecimento lexical adquirido por meio da leitura pode afetar o reconhecimento de palavras (PERFETTI; HART, 2001).

O estudo de Artieda (2007) procurou responder se a literacia e os hábitos de leitura na primeira língua influenciariam o aprendizado da L2 de adultos com variados níveis de proficiência. Conforme os resultados, a autora sugere que a literacia na L1 pode agir como um limite mínimo para que o aprendizado de uma L2 ocorra e que a habilidade de soletrar na L1 pode ser importante para o aprendizado da L2 até mesmo para aprendizes com proficiência intermediária. Além disso, no caso dos aprendizes com proficiência avançada, os hábitos de leitura na L2 parecem ter promovido o aprendizado da L2 mais intensamente do que a habilidade de soletrar na L1. Esse estudo demonstra que a habilidade de soletração pode ter mais impacto no aprendizado de uma L2 para aprendizes menos proficientes e que, no caso dos mais proficientes, os hábitos de leitura apresentam uma influência maior. Assim, a experiência de leitura pode ter impacto no conhecimento lexical e, consequentemente, no aprendizado de uma L2.

Os estudos mencionados acima sugerem que a experiência de leitura - hábitos de leitura e também exposição a materiais escritos - influencia o reconhecimento de palavras e o desenvolvimento de vocabulário tanto na L1 quanto na L2. Por outro lado, é preciso lembrar que os bilíngues estão expostos a duas línguas e que o tempo de imersão em uma língua é inversamente proporcional ao tempo de imersão em outra. Nesse sentido, o estudo de Whitford e Titone (2015) apresenta evidência em favor da Hipótese do Atraso de Frequência (HQF, Frequency-Lag Hypothesis; 
GOLLAN; SLATTERY; GOLDENBERG; VAN ASSCHE; DUYCK; RAYNER, 2011). Essa abordagem afirma que, já que bilíngues precisam dividir o uso de língua entre duas línguas, eles acabam usando-as individualmente com menos frequência se comparados a monolíngues. Consequentemente, bilíngues utilizam itens lexicais específicos de uma língua com menos frequência do que monolíngues. Em outras palavras, o efeito de frequência tem um impacto maior para bilíngues e produz uma desvantagem maior durante o acesso a palavras de baixa frequência (GOLLAN et al., 2011).

Em consonância tanto com a HAF quanto com a HQL, Whitford e Titone (2015) examinaram a influência da exposição atual à L2 na habilidade de leitura em L1 e em L2. Participantes bilíngues inglês-francês e francês-inglês realizaram uma tarefa de leitura de frases curtas, e seus movimentos oculares foram registrados. Os resultados demonstraram que a exposição atual à L2 modulou a fluência de leitura e a janela de percepção dos participantes. Os bilíngues que relataram estar mais expostos à L2 apresentaram maior fluência de leitura na L2 mas menor na L1; também foram mais influenciados pela diminuição do número de caracteres perceptíveis durante a leitura das frases em L2 mas não em L1. Isso corrobora com as ideias da HAF e da HQL no sentido de que maior exposição à L2 pode atuar em detrimento da qualidade lexical na L1 (WHITFORD; TITONE, 2015), já que ambas dependem de frequência.

Ainda em sintonia com a HQL e a HQF, Rudell e Hu (2010) demonstraram que monolíngues de inglês respondem mais rapidamente a palavras e apresentam latências de potenciais relacionados a eventos (PREs) mais curtas do que bilíngues chinês-inglês. Os participantes monolíngues tinham mais experiência com palavras em inglês e estavam mais expostos a elas do que os bilíngues, fato esse que parece explicar os resultados. De acordo com a HQL, a maior experiência com as palavras de uma língua pode facilitar o seu reconhecimento. Esse parece ser o caso, já que os monolíngues, por utilizarem apenas uma língua, estavam expostos ao inglês por maiores períodos de tempo. Além disso, conforme a Hipótese do Atraso de Frequência, bilíngues terão menos experiência com determinada língua em comparação com monolíngues por estarem expostos a duas línguas.

A experiência de leitura e a exposição a uma língua influenciam as representações lexicais de monolíngues e de bilíngues. No caso dos bilíngues, há ainda uma diferença de experiência linguística devido ao uso dividido de cada uma das línguas. Assim, é importante pensar em suas habilidades linguísticas e em como elas podem ser medidas.

\subsection{Habilidades linguísticas}

Habilidades linguísticas, assim como as línguas, são complexas. É possível teorizar que elas formem um sistema composto de múltiplas dimensões (PENTIMONTI et al., 2015). Geralmente, elas são divididas por modalidade - compreensão e produção - ou de acordo com dois grande aspectos linguísticos - léxico e sintaxe (PENTIMONTI et al., 2015). Mais especificamente, em contextos de ensino de línguas, elas costumam ser separadas em modalidade oral e escrita: compreensão de fala e de leitura e produção de fala e de escrita.

Essas quatro habilidades podem ser avaliadas com base em testes padronizados desenvolvidos especificamente para medir os conhecimentos envolvidos e seu uso. Alguns exemplos desses testes são o IELTS, o TOEFL e o Cambridge English Proficiency. Além disso, há a possibilidade de uma autoavaliação ser realizada por meio de questionários. Geralmente, esses instrumentos são questionários de histórico de uso de língua e incluem perguntas referentes a idade de aquisição da língua, contextos de uso e autopercepção sobre as habilidades linguísticas e a proficiência. Dois exemplos dessas medidas são o Questionário de Histórico de Linguagem (Language History Questionnaire, LI et al., 2014) e o Questionário de Proficiência e Experiência de Língua (Language Experience and Proficiency Questionnaire, MARIAN; BLUMENFELD; KAUSHANSKAYA, 2007). 
Apesar de se tratarem de medidas subjetivas e sujeitas a falhas de autopercepção e de recordação de memórias do participante ao preencher o questionário, esses instrumentos de autoavaliação são confiáveis para uso em pesquisas sobre habilidades linguísticas. Scholl, Finger e Arêas da Luz Fontes (2017) apontam que os participantes são capazes de relatar suas habilidades e experiências com as línguas faladas de forma consistente. Além disso, Scholl (2016) demonstrou que as respostas dadas ao Questionário de Experiência e Proficiência Linguística (QuExPLi) estavam positiva e moderadamente correlacionadas à pontuação desses participantes no teste padronizado TOEFL ITP. Dessa forma, é possível afirmar que questionários de histórico de uso de língua, especialmente aqueles que são amplamente utilizados e testados, são instrumentos confiáveis e válidos para medir as habilidades linguísticas de participantes.

Além disso, parece haver bastantes evidências na literatura de que há relações entre leitura e escrita. Wong (2018) aponta que estudos demonstraram haver correlações entre os processos de leitura e escrita nos mais diversos níveis linguísticos. Em sua investigação longitudinal, Wong (2018) observou que a habilidade de escrita de frases em chinês de jovens aprendizes estava fortemente correlacionada à sua habilidade de leitura de um ano antes e à sua atual leitura de frases. Esse estudo sugere a associação entre as habilidades de leitura e de escrita em uma língua e também indica vinculação com os hábitos de leitura desses participantes. Paralelamente, Parodi (2007) também apresenta correlações positivas e fortes entre medidas de leitura e de escrita, as quais eram mais fortes para níveis mais globais de textos (super e macroestrutura) e moderadas para níveis mais detalhados (microestrutura).

Considerando novamente o caso do bilinguismo, Lee e Schallert (2016) explicam que o aprendizado de uma segunda língua é influenciado pelas habilidades de leitura e de escrita. Nesse sentido, hábitos de leitura podem beneficiar as habilidades de leitura e de escrita da mesma forma que as atividades de escrita podem aprimorar as habilidades de leitura e de escrita - especialmente em conjunto a estratégias. As autoras também comentam que é possível que esses ganhos sejam modulados pela proficiência do falante nessa língua.

Esses elos entre hábitos de leitura e habilidades linguísticas, tanto em uma língua quanto em duas, parecem permitir influências bidirecionais. Ademais, podem impactar o reconhecimento de palavras, incluindo interações entre línguas que ocorrem com pessoas bilíngues.

\subsection{Acesso lexical bilíngue}

A investigação sobre o acesso lexical bilíngue se debruça sobre os momentos iniciais do processamento e do reconhecimento de palavras, seja por meio de tarefas com palavras isoladas ou inseridas em contextos frasais. Em especial, o foco está na potencial interação entre as línguas dos bilíngues, nos aspectos que a influenciam e nas suas consequências para o processamento da linguagem.

Há ampla evidência de que as representações lexicais de ambas as línguas de um bilíngue estão sempre ativadas durante o reconhecimento de palavras. Em outras palavras, estudos mostram que bilíngues não são capazes de desativar uma das línguas durante a leitura (DIJKSTRA; GRAINGER; VAN HEUVEN, 1999; DE BRUIJN ET AL., 2001; BRENDERS; VAN HELL; DIJKSTRA, 2011; POARCH; VAN HELL, 2014; AREAS DA LUZ FONTES; SCHWARTZ, 2015; BARCELOS, 2016). Isso significa que, diferente dos monolíngues, cujas representações lexicais remetem a apenas uma língua, bilíngues coativam as representações de um item lexical de ambas as línguas durante o acesso lexical. Esse fenômeno é descrito pela hipótese da não seletividade da língua, que afirma que não é possível para o bilíngue selecionar uma única língua durante o acesso lexical (DIJKSTRA; VAN HEUVEN, 2002; VAN HEUVEN; DIJKSTRA, 2010; LAGROU; HARTSUIKER; DUYCK, 2015).

Efeitos da coativação linguística podem ser detectados utilizando itens lexicais que compartilhem aspectos linguísticos entre línguas, tanto na L1 quanto na L2 (SCHWARTZ; 
KROLL; DIAZ, 2007). Palavras podem compartilhar características ortográficas, fonológicas e/ou semânticas tanto dentro de uma mesma língua quanto entre línguas diferentes. Por exemplo, homófonos interlinguais apresentam sobreposição na sua pronúncia, homógrafos interlinguais se sobrepõem ortograficamente, e homônimos interlinguais compartilham ortografia e significado - e às vezes inclusive o status ambíguo. Palavras cognatas também são usadas, pois tendem a compartilhar quase todos esses aspectos em variados níveis, geralmente com maior sobreposição de significado e ortografia do que de pronúncia. Similarmente, esses itens lexicais também podem refletir diferenças de qualidade lexical para as representações de cada uma das línguas (PERFETTI; HART, 2001).

Essas representações coativadas competem por maior ativação, e a quantidade de sobreposição de representações ortográficas e fonológicas entre línguas pode influenciar o nível de competição entre os itens (SCHWARTZ; KROLL; DIAZ, 2007). É possível que esses efeitos sejam modulados pelo nível de proficiência na L2 (BRENDERS; VAN HELL; DIJKSTRA, 2011; FRIESEN; JARED; HAIGH, 2014; PIVNEVA; MERCIER; TITONE, 2014; DURLIK et al., 2016), e eles ocorrem até mesmo durante a leitura silenciosa (CARRASCO-ORTIZ; MIDGLEY; FRENCK-MESTRE, 2012).

O estudo do acesso lexical bilíngue pode contribuir para a compreensão do processamento da língua, de como esse mesmo léxico está organizado e de quais fatores podem influenciar o reconhecimento das palavras. No caso específico da leitura, vale ressaltar que os diferentes sistemas de escrita não são impecáveis na representação de todos os aspectos das línguas, evitando qualquer ambiguidade (KESSLER; TREIMAN, 2015). Pelo contrário, mapeamentos um-para-um não são a regra. É aqui que investigar o acesso lexical pode esclarecer como os conhecimentos sobre a língua e as palavras influenciam o seu processamento. A ativação simultânea de duas representações ortográficas, fonológicas e ou semânticas podem competir entre si e atrasar o acesso lexical, ou reforçar uma ativação e acelerar o processo. E, quando tratamos de bilíngues, esses fenômenos de competição e de reforço ocorrem tanto dentro de uma língua quanto entre elas. Essa interação deve ser considerada ao se trabalhar com acesso lexical bilíngue.

\section{Método}

\subsection{Objetivo e hipóteses}

O objetivo deste estudo é investigar as relações entre a experiência de leitura em L2, as habilidades autoavaliadas de leitura e de escrita na L2 e o acesso lexical na L2 de bilíngues português-inglês.

As hipóteses são de que as correlações encontradas irão confirmar o que as teorias apresentadas acima propõem, isto é, experiência de leitura em L2 estará relacionada às habilidades de leitura e de escrita e ao acesso lexical na L2, as habilidades de leitura e de escrita estarão relacionadas ao acesso lexical na L2 e as habilidades de leitura e de escrita estarão relacionadas entre si.

\subsection{Desenho}

Os construtos de interesse neste estudo são: o acesso lexical na L2 (inglês), operacionalizado pelas variáveis tempo de reação e taxa de erro na tarefa de decisão de significados; a experiência de leitura na L2, operacionalizada pela variável dos hábitos de leitura em L2; e as habilidades linguísticas na L2, operacionalizadas pelas variáveis de habilidades de escrita e de leitura em L2 autorrelatadas. 
Este é um estudo correlacional, em que a relação entre o desempenho em uma tarefa de decisão de significado e os hábitos de leitura em L2 e as habilidades de escrita e de leitura em L2 será analisada.

\subsection{Participantes}

Inicialmente, participaram voluntariamente deste estudo 84 (21 homens) estudantes universitários, cuja idade média era 23,3 anos ( $D P=5,73$, de 17 a 49 anos). Eles foram selecionados de acordo com o critério de que deveriam falar o português brasileiro como L1 e o inglês como L2; caso falassem uma terceira língua, a proficiência nessa língua deveria ser inferior que a em inglês ${ }^{21}$. Os dados de duas participantes foram descartados, pois uma delas relatou ter proficiência em alemão maior que em inglês, e a outra desistiu da participação. Assim, os dados de 82 participantes foram utilizados. O Quadro 1 apresenta um resumo do seu histórico de uso de língua.

Quadro 1 - Alguns aspectos do histórico de uso de língua dos participantes.

\begin{tabular}{|c|c|}
\hline Total de anos usando inglês & $13,71(\mathrm{DP}=6,4)$ \\
\hline Média da idade em que começou a ouvir em inglês & $8,51(\mathrm{DP}=3,65$, de 1 a 19$)$ \\
\hline Média da idade em que começou a falar em inglês & $11 \operatorname{anos}(\mathrm{DP}=4,14$, de 2 a 26$)$ \\
\hline Média da idade em que começou a ler em inglês & $10,56 \operatorname{anos}(\mathrm{DP}=3,01$, de 4 a 18$)$ \\
\hline Média da idade em que começou a escrever em inglês & $11,21 \operatorname{anos}(\mathrm{DP}=3,26$, de 4 a 21$)$ \\
\hline Média de habilidade de aprendizado de língua & $\begin{array}{l}5,13(\mathrm{DP}=0,97 ; 1=\text { muito fraco, } 7= \\
\text { excelente })\end{array}$ \\
\hline Moda da habilidade atual de escuta & $6(1=$ muito fraco, $7=$ quase nativo $)$ \\
\hline Moda da habilidade atual de fala & $6(1=$ muito fraco, $7=$ quase nativo $)$ \\
\hline Moda da habilidade atual de leitura & $6(1=$ muito fraco, $7=$ quase nativo $)$ \\
\hline Moda da habilidade atual de escrita & $5(1=$ muito fraco, $7=$ quase nativo $)$ \\
\hline $\begin{array}{l}\text { Moda de horas diárias lendo por prazer, para a escola ou trabalho e ouvindo } \\
\text { o rádio em inglês }\end{array}$ & 1 hora por dia \\
\hline Moda de horas diárias vendo TV em inglês & 2 horas por dia \\
\hline Moda de horas diárias calculando em inglês & 1 hora por dia \\
\hline Moda de horas diárias lembrando números em inglês & 2 horas por dia \\
\hline Moda de horas diárias sonhando em inglês & 3 horas por dia \\
\hline $\begin{array}{l}\text { Moda de horas diárias expressando emoções, pensando e falando sozinho } \\
\text { em inglês }\end{array}$ & 5 horas por dia \\
\hline
\end{tabular}

\footnotetext{
${ }^{21}$ Dos 82 participantes finais, $53(64,63 \%)$ relataram ter ao menos conhecimento básico de outra língua além do português brasileiro e do inglês. Trinta e três deles mencionaram conhecer espanhol; onze, francês; seis, alemão; cinco, italiano; cinco, japonês; três, chinês; dois, latim; um, flamengo; um, russo; e um, hindi.
} 


\subsection{Instrumentos}

\subsubsection{Questionário de hábitos de leitura e experiência de leitura em L2}

Este questionário é o mesmo utilizado por Pratheeba e Krashen (2013) para medir com que frequência os participantes realizavam atividades de leitura em inglês, sua L2, e quais materiais eram lidos nessa língua. Ele era composto de vinte perguntas sobre hábitos de leitura, as quais envolviam tanto materiais impressos quanto digitais, diferentes contextos de leitura e diversos gêneros textuais. As respostas eram estruturadas em uma escala de seis pontos: zero equivalia a "nunca" e 5, a "sempre". Exemplos das perguntas seguem nos excertos (1) e (2).

(1) 1. Are you in the habit of reading daily newspapers in English?
( ) 0
( ) 1
( ) 2
( ) 3
( ) 4
( ) 5

(2) 5 . Are you in the habit of reading short stories in English?
( ) 0
( ) 1
( ) 2
( ) 3
( ) 4 ( ) 5

Os participantes responderam o questionário em uma folha de papel. As respostas numéricas para as vinte perguntas foram somadas, e a pontuação final (máximo 100) foi definida como a experiência de leitura em L2 dos participantes.

\subsubsection{Habilidades linguísticas autoavaliadas}

As habilidades linguísticas em leitura e em escrita dos participantes foram registradas a partir dos itens específicos do questionário de histórico de uso de língua amplamente utilizado, criado por Li, Zhang, Tsai e Puls (2014). As pontuações de habilidade foram relatadas pelos próprios participantes com base em uma escala de sete pontos, na qual 1 equivalia a "muito fraca" e 7, a "quase nativa". Este questionário foi preenchido no computador.

\subsubsection{Tarefa de decisão de significado}

Esta tarefa foi construída seguindo descrições publicadas por Perfetti e Hart (2001) e contém apenas estímulos em inglês. Ela instrui o participante a ler uma palavra-prime que aparecerá por $300 \mathrm{~ms}$ na tela do computador, em seguida, ler uma palavra-alvo que aparecerá por até $3000 \mathrm{~ms}$ e, então, decidir se a palavra-alvo está relacionada semanticamente à palavra-prime usando as teclas (L) para "sim" e (A) para "não". Esta tarefa permite medir o tempo de reação dos participantes durante o acesso ao significado dos itens lexicais em questão. Os estímulos eram 64 palavras-prime, nenhuma das quais era cognata entre português e inglês ou ambígua. Todas as palavras-prime eram pareadas a palavras-alvo, que poderiam ser relacionadas semanticamente ou não. Além disso, 32 palavras distratoras foram adicionadas com palavras-alvo não relacionadas. Os pares de palavras foram organizados em listas para contrabalancear as palavras-alvo relacionadas e não relacionadas.

Os estímulos foram selecionados de acordo com os seguintes critérios. As palavras-prime foram comparadas aos estímulos ambíguos utilizados por Arêas da Luz Fontes e Schwartz (2015), por Armstrong, Tokowicz e Plaut (2012) e por Maciejewski and Klepousniotou (2016) para que não fossem ambíguas. Foram excluídas as palavras com frequência menor que 10.0 conforme o CELEX2 (BAAYEN; PIEPENBROCK; GULIKERS, 1995), empréstimos linguísticos em português (p. ex., hertz, jeans) e palavras polissêmicas (p. ex., coat, que pode significar casaco ou cobrir). O grau de polissemia das palavras foi avaliado com o auxílio do site Wordsmith. Todas as palavras- 
prime foram controladas em função de frequência, número de letras e categoria gramatical ${ }^{22}$. As palavras-alvo foram selecionadas seguindo as Normas de Associação Livre da Universidade do Sul da Flórida, que é um corpus de dados de associação livre para 72.000 de pares de palavras do inglês (NELSON; MCEVOY; SCHREIBER, 2004; NELSON; MCEVOY; SCHREIBER, 1998, appendix A).

\subsection{Procedimentos}

Após assinarem o Termo de Consentimento Livre e Esclarecido, os participantes sentavam-se na frente de um computador na sala do grupo de pesquisa. Eles realizavam a tarefa de decisão de significado primeiro por ser a mais cansativa. Em seguida, preenchiam o questionário de hábitos de leitura e experiência de leitura na L2 em papel e o questionário de histórico de uso de língua no computador, ou vice-versa. Por fim, agradecemos a sua participação voluntária.

\section{Resultados}

Os dados coletados foram compilados em planilhas juntamente às respostas numéricas dos participantes aos questionários. As seguintes variáveis foram selecionadas para operacionalizar os construtos que estavam sendo investigados. O tempo de reação médio para as palavras-alvo relacionadas (1a) e não relacionadas (2a) às palavras-prime e a porcentagem de erro para as palavrasalvo relacionadas (3a) e não relacionadas (4a) às palavras-prime representariam o acesso lexical a itens da L2. As pontuações das habilidades de leitura (5a) e de escrita (6 ${ }^{a}$ ) na L2 autoavaliadas equivaleriam ao nível dessas habilidades dos participantes. E a soma da pontuação das respostas ao questionário de hábitos de leitura e experiência de leitura em L2 (7a) refletiria o grau de experiência de leitura desses participantes em sua L2.

Foram realizados testes de normalidade nessa amostra de dados e concluiu-se que suas distribuições eram próximas à normal. Assim, foram realizadas correlações de Pearson, cruzando as sete variáveis mencionadas acima. De um modo geral, as correlações encontradas foram negativas e positivas, apresentando força de fraca $(0,20$ a 0,39) a moderada $(0,40$ a 0,59) (EVANS, 1996).

O tempo de reação médio para as palavras-alvo relacionadas semanticamente às palavrasprime apresentou correlação negativa tanto com a habilidade de leitura, $r=-0,275, p=0,012$, quanto com a habilidade de escrita, $r=-0,338, p=0,002$. Da mesma forma, a porcentagem de erro para as palavras-alvo relacionadas semanticamente às palavras-prime apresentou correlação negativa tanto com a habilidade de leitura, $r=-0,29, p=0,008$, quanto com a habilidade de escrita, $r=$. $0,293, p=0,008$. Isso indica que as habilidades de leitura e de escrita na L2 estão negativa e fracamente correlacionadas ao tempo de reação e à porcentagem de erro dos participantes ao decidirem se pares de palavras da L2 são semanticamente relacionados, confirmando as nossas hipóteses. Ou seja, quanto melhor os participantes se autoavaliavam nas habilidades de leitura e de escrita na L2, mais rapidamente eles decidiam se a palavra-alvo era relacionada semanticamente à palavra-prime.

Além disso, foi possível verificar que houve correlação positiva entre a experiência de leitura em L2 e a habilidade de leitura, $r=0,283, p=0,010$, e de escrita na L2, $r=0,253, p=0,022$. Essa correlação, apesar de fraca, demonstra que parte da variação nas habilidades de leitura e de escrita na L2 é compartilhada com a experiência de leitura nessa língua, o que também confirma nossas hipóteses.

\footnotetext{
${ }^{22} \mathrm{O}$ número de sílabas não foi controlado, já que a estrutura da sílaba costuma variar de uma língua para outra (AKMAJIAN; DEMERS; FARMER; HARNISH, 2010, p. 127). Doutro modo, optamos por controlar o número de letras.
} 
Entretanto, não houve correlação significativa (todos os p > 0,08) entre os tempos de reação ou as porcentagens de erro na tarefa de decisão de significados e a experiência de leitura em L2. Este resultado não confirma as nossas hipóteses e diverge do que seria esperado conforme a literatura apresentada.

Por fim, as habilidades de leitura e de escrita na L2 demonstraram estar correlacionadas positiva e moderadamente uma com a outra, $r=0,505, p<0,001$. Isso sugere que as pontuações autoavaliadas pelos participantes para suas habilidades de escrita e de leitura na L2 estão relacionadas mais proximamente, também confirmando nossas hipóteses.

\section{Discussão}

De forma geral, os resultados observados neste estudo podem ser explicados pelas teorias e hipóteses apresentadas na seção de referencial teórico. As correlações negativas e fracas entre os tempos de reação e as porcentagens de erro no acesso lexical na L2 e a habilidade autoavaliada de leitura na L2 estão de acordo com as previsões da HQL de Perfetti e Hart (2001, 2002), em especial relativamente à automatização do reconhecimento de palavras causada por uma qualidade lexical maior. Em outras palavras, isso evidencia que hábitos de leitura mais frequentes na L2 contribuem para aumentar o conhecimento lexical do leitor nessa língua, os quais promovem uma leitura mais rápida e eficiente. A pouca força dessas correlações pode estar ligada à exposição dos participantes às L1 e L2. A maioria deles era estudante universitário em um contexto em que a L1 era predominante. Apesar de muitos deles cursarem disciplinas realizadas na L2 e/ou trabalharem com o inglês, o português ainda predominava. Nesse sentido, a exposição equivalente ou maior à L1 pode ter influenciado a força das correlações, o que estaria em consonância com a HAF (GOLLAN et al., 2011).

Similarmente, a correlação positiva e fraca entre experiência de leitura em L2 e a habilidade autoavaliada de leitura em L2 é compatível tanto com a HQL quanto com a HAF. A leitura mais frequente na L2 irá aprimorar o conhecimento sobre as representações nessa língua e, consequentemente, a habilidade de leitura; essa melhora pode ser sentida pelo leitor e refletida em um questionário de histórico de uso de língua (SCHOLL, 2016).

Quanto à suposição de que habilidades de escrita também parecem estar relacionadas à leitura, foi possível observar evidências disso. As correlações negativas e fracas entre os tempos de reação e a porcentagem de erro no acesso lexical na L2 e a habilidade autoavaliada de escrita na L2 estão em consonância com os resultados apresentados por Parodi (2007), Lee e Schallert (2016) e Wong (2018). Porém, a diferença de força das relações pode ser explicada por fatores como idade média da amostra e contexto de língua. Os participantes de Wong (2018) eram estudantes de 11 a 12 anos de minorias étnicas e estavam aprendendo chinês e inglês como L2 na escola, todas as tarefas foram realizadas em chinês. Os participantes de Parodi (2007) eram também estudantes de aproximadamente 13 anos, mas todas as tarefas foram realizadas na sua L1, espanhol chileno. Por outro lado, os participantes deste estudo eram estudantes universitário de idade média de 23 anos de forma que esses 10 ou mais anos de diferença entre as amostras podem estar influenciando os resultados. Além disso, esta investigação selecionou bilíngues português-inglês, o que, deixando o fator idade de lado, aproxima-a à de Wong (2018). Porém, Parodi (2007) utilizou monolíngues, uma diferença que deve ser mencionada. Por fim, Lee e Schallert (2016) não realizaram testes de correlação em seu estudo, mas seus participantes também eram estudantes de 13 a 14 anos que falavam coreano como L1 e estavam aprendendo inglês como L2 na escola; todas as tarefas foram realizadas em inglês. Elas sugerem que, ao longo do tempo, a leitura em L2 pode beneficiar a escrita em L2 e vice-versa. Assim, consideradas as diferenças entre métodos dos estudos citados acima, é possivel indicar alguma relação entre a habilidade de escrita em L2 e a velocidade e precisão de leitura em L2. 
Um resultado análogo aos recém discutidos é a correlação positiva e fraca entre experiência de leitura na L2 e a habilidade autoavaliada de escrita na L2. Os benefícios vistos na habilidade de escrita dos participantes de Lee e Schallert (2016) podem ser ao menos parcialmente atribuídos à sua maior frequência de leitura na L2. Vale ressaltar que as autoras avisam que outros fatores podem ter colaborado para essa melhora os quais não puderam ser controlados no estudo, como diferentes atividades escolares dos estudantes. Da mesma forma, a correlação fraca aqui observada indica que as variáveis em questão explicam apenas uma pequena parte da variância nos dados coletados. Parece haver uma relação, mas ela não dá conta de toda a variação observada.

Diferentemente do que havia sido previsto nas hipóteses a partir da literatura, não houve correlação significativa entre os tempos de reação e a porcentagem de erro na tarefa de decisão de significado e a experiência de leitura em L2. Esse resultado surpreende pois é contrário ao encontrado na literatura. No entanto, é possível que questões metodológicas possam ajudar a explicar a ausência da correlação esperada. Uma possibilidade diz respeito ao questionário de hábitos de leitura utilizado na pesquisa. O questionário mede a frequência com que os participantes leem materiais de diversos gêneros textuais - talvez fosse necessária também uma medida de quantidade de material lido dentro da frequência reportada. Além disso, as palavras utilizadas na tarefa de decisão de significados podem ter sido pouco familiares para os participantes da pesquisa. Em estudos posteriores seria necessária uma checagem pós-teste para averiguar tal possibilidade.

Finalmente, foi encontrado que as habilidades de leitura e de escrita na L2 estavam correlacionadas positiva e moderadamente uma com a outra. Esse resultado está de acordo com Wong (2018) e Parodi (2007). Porém, pensando nas diferenças de idade entre as amostras, pode ser sensato cogitar que essa correlação tenha sido inflada no momento do relato das habilidades. Essas medidas linguísticas não estão representando um mesmo construto, pois, nesse caso, equivaleriam a uma correlação quase perfeita. Porém, é possível que os participantes tenham definido as habilidades de leitura e de escrita seguindo critérios similares, o que pode ter levado a alguma sobreposição na variável aqui estudada. Assim, 25\% da variância de uma habilidade é compartilhada com a outra habilidade, o que sugere que uma possa influenciar parcialmente a outra. Esse valor pode estar um pouco inflado; contudo, ele de fato corrobora resultados de outros estudos e contribui para demonstrar a influência de uma habilidade linguística em outras.

\section{Conclusão}

Este estudo investigou as relações entre a experiência de leitura em L2, as habilidades autoavaliadas de leitura e escrita em L2 e o acesso lexical em L2 de bilíngues português-inglês. Foram observadas correlações de fracas a moderadas entre esses construtos, com exceção de uma correlação não significativa inesperada. $\mathrm{O}$ acesso lexical em L2 apresentou correlações negativas fracas com as habilidades de leitura e de escrita em L2. A experiência de leitura em L2 demonstrou estar correlacionada positiva e fracamente com as habilidades de leitura e de escrita. Não houve correlação entre a experiência de leitura em L2 e o acesso lexical em L2, o que pode ter sido causado por questões metodológicas já que as evidências na literatura são presentes. Por fim, as habilidades de leitura e de escrita correlacionaram positiva e moderadamente entre si. Esses resultados indicam que, para esta amostra de estudantes universitários bilíngues, os hábitos de leitura em inglês, sua autopercepção das habilidades de leitura e de escrita e sua velocidade e precisão ao decidirem se palavras em inglês são relacionadas semanticamente estão parcialmente relacionadas.

Estes achados contribuem para a discussão sobre as implicações da leitura e a sua importância. É possível afirmar que hábitos de leitura frequentes em uma L2 são benéficos tanto para o conhecimento lexical quanto para as habilidades de leitura e escrita nessa língua. Esse aprimoramento pode depender de tempo e de outros fatores não controlados aqui para mostrar 
56 | Pietra Cassol Rigatti, Ana Beatriz Arêas da Luz Fontes

um efeito mais imediato. Apesar disso, a recomendação de que ler faz bem para o aprendizado de uma L2 parece ainda ser positiva.

\section{Referências}

AKMAJIAN, A.; DEMERS, R. A.; FARMER, A. K.; HARNISH, R. M. Linguistics: An Introduction to Language and Communication. 6th ed. Massachusetts: The MIT Press, 2010.

AREAAS DA LUZ FONTES, A. B.; SCHWARTZ, A. I. Bilingual access of homonym meanings: Individual differences in bilingual access of homonym meanings. Bilingualism: Language and Cognition, v. 18, n. 4, 2015, pp. 639-656.

ARTIEDA, G. The role of L1 literacy and reading habits on the L2 achievement of adult learners of English as a foreign language. System, n. 66, 2017, pp. 168-176.

BARCELOS, L. O acesso lexical em trilingues brasileiros falantes de português, inglês e francês. Dissertação de Mestrado: Universidade Federal do Rio Grande do Sul, 2016.

BRENDERS, P.; VAN HELL, J. G.; DIJKSTRA, T. Word recognition in child second language learners: Evidence from cognates and false friends. Journal of Experimental Child Psychology, n. 109, 2011, pp. 383-396.

CARRASCO-ORTIZ, H.; MIDGLEY, K. J.; FRENCK-MESTRE, C. Are phonological representations in bilinguals language specific? An ERP study on interlingual homophones. Psychophysiology, n. 49, 2012, pp. 531-543.

DE BRUIJN, E. R. A.; DIJKSTRA, T.; CHWILlA, D. J.; SCHRIEFERS, H. J. Language context effects on interlingual homograph recognition: evidence from event-related potentials and response times in semantic priming. Bilingualism: Language and Cognition, v. 4, n. 2, 2001, pp. 155168.

DIJKSTRA, T.; GRAINGER, J.; VAN HEUVEN W. J. B. Recognition of Cognates and Interlingual Homographs: The Neglected Role of Phonology. Journal of Memory and Language, v. 41, 1999, pp. 496-518.

DIJKSTRA, T.; VAN HEUVEN, W. J. B. The architecture of the bilingual word recognition system: From identification to decision. Bilingualism: Language and Cognition, v. 5, n. 3, 2002, pp. 175-197.

DURLIK, J.; SZEWCZYK, J.; MUSZYŃSKI, M.; WODNIECKA, Z. Interference and Inhibition in Bilingual Language Comprehension: Evidence from Polish-English Interlingual Homographs. PLoS ONE, v. 11, n. 3, 2016.

FRIESEN, D. C.; JARED, D.; HAIGH, C. A. Phonological Processing Dynamics in Bilingual Word Naming. Canadian Journal of Experimental Psychology, v. 68, n. 3, 2014, pp. 179-193.

GOLLAN, T. H.; SLATTERY, T. J.; GOLDENBERG, D.; VAN ASSCHE, E.; DUYCK, W.; RAYNER, K. Frequency Drives Lexical Access in Reading but not in Speaking: The Frequency-Lag Hypothesis. Journal of Experimental Psychology: General, v. 140, n. 2, 2011, pp. 186-209.

HAMADA, M.; KODA, K. Influence of First Language Orthographic Experience on Second Language Decoding and Word Learning. Language Learning, v. 58, n. 1, 2008, pp. 1-31.

HAMILTON, S.; FREED, E.; LONG, D. L. Word-Decoding Skill Interacts With Working Memory Capacity to Influence Inference Generation During Reading. Reading Research Quarterly, v. 51, n. 4, 2016, pp. 391-402.

JARED, D.; KROLL, J. F. Do Bilinguals Activate Phonological Representations in One or Both of Their Languages When Naming Words? Journal of Memory and Language, v. 44, 2001, pp. 2-31.

KESSLER, B.; TREIMAN, R. Writing Systems: Their Properties and Implications for Reading. In: POLLATSEK, A.; TREIMAN, R. (Eds.) The Oxford Handbook of Reading. Editor chefe Perter E. Nathan. Oxford University Press, 2015. 
LAGROU, E.; HARTSUIKER, R. J.; DUYCK, W. Do Semantic Sentence Constraint and L2 Proficiency Influence Language Selectivity of Lexical Access in Native Language Listening? Journal of Experimental Psychology: Human Perception and Performance, v. 41, n. 6, 2015, pp. 1524-1538.

LEE, J.; SCHALLERT, D. Exploring the Reading-Writing Connection: A Yearlong ClassroomBased Experimental Study of Middle School Students Developing Literacy in a New Language. Reading Research Quarterly, v. 51, n. 2, 2015, pp. 143-164.

LI, P.; ZHANG, F.; TSAI, E.; PULS, B. Language history questionnaire (LHQ 2.0): A new dynamic web-based research tool. Bilingualism: Language and Cognition, v. 17, n. 3, 2014, pp. 673680.

MARIAN, V.; BLUMENFELD, H. K.; KAUSHANSKAYA, M. The Language Experience and Proficiency Questionnaire (LEAP-Q): assessing language profiles in bilinguals and multilinguals. Journal of Speech, Language, and Hearing Research, v. 50, n. 4, 2007, pp. 940-967.

NELSON, D. L.; MCEVOY, C. L.; SCHREIBER, T. A. The University of South Florida word association, rhyme, and word fragment norms. <http://www.usf.edu/FreeAssociation/. 1998, Appendix A>.

NELSON, D. L.; MCEVOY, C. L.; SCHREIBER, T. A. The University of South Florida free association, rhyme, and word fragment norms. Behavior Research Methods, Instruments, $\mathcal{E}$ Computers, v. 36, n. 3, 2004, pp. 402-407.

PARODI, G. Reading-writing connections: Discourse-oriented research. Reading and Writing, v. 20, 2007, pp. 225-250.

PERFETTI, C. A.; HART, L. The Lexical Bases of Comprehension Skill. In: GORFIEN, D. S. (Ed.) On the consequences of meaning selection: Perspectives on resolving lexical ambiguity. Washington, DC: American Psychological Association, 2001, pp. 67-86.

PERFETTI, C. A.; HART, L. The lexical quality hypothesis. In: VERHOEVEN, L.; ELBRO, C. REITSMA, P. (Eds.) Precursors of Functional Literacy. Studies in Written Language and Literacy, v. 11, 2002, pp. 189-213.

PERFETTI, C. Reading Ability: Lexical Quality to Comprehension. Scientific Studies of Reading, v. 11, n. 4, 2007, pp. 357-383.

PIVNEVA, I.; MERCIER, J.; TITONE, D. Executive control modulates cross-language lexical activation during L2 reading: evidence from eye movements. Journal of Experimental Psychology: Learning, Memory, and Cognition, v. 40, n. 3, 2014, pp. 787-796.

POARCH, G. J.; VAN HELL, J. G. Cross-language activation in same-script and different-script trilinguals. International Journal of Bilingualism, v. 18, n. 6, 2014, pp. 693-716.

PRATHEEBA, N.; KRASHEN, S. Self-reported reading as a predictor of vocabulary knowledge. Perceptual and Motor Skills, v. 117, n. 2, 2013, pp. 442-448.

SANTOS-DÍAZ, I. The influence of reading on the vocabulary in mother tongue and foreign language. Ocnos, v. 16, n. 1, 2017, pp. 79-88.

SCHOLL, A. P. Proficiência autoavaliada através de um questionário de histórico da linguagem. Dissertação de Mestrado: Universidade Federal do Rio Grande do Sul, 2016.

SCHOLL, A. P.; FINGER, I.; AREAS DA LUZ FONTES, A. B. Fatores de experiência linguística associados à proficiência autoavaliada por usuários de inglês como língua adicional. Letrônica, v. 10, n. 2, 2017, pp. 689-699.

SCHWARTZ, A. I.; KROLL, J. F.; DIAZ, M. Reading words in Spanish and English: Mapping orthography to phonology in two languages. Language and Cognitive Processes, v. 22, n. 1, 2007, pp. 106-129.

TAYLOR, J. N.; PERFETTI, C. A. Eye movements reveal readers' lexical quality and reading experience. Reading and Writing, v. 29, 2016, pp. 1069-1103.

VAN HEUVEN, W. J. B.; DIJKSTRA, T. Language comprehension in the bilingual brain: fMRI and ERP support for psycholinguistic models. Brain Research Review, v. 64, 2010, pp. 104-122. 
58 | Pietra Cassol Rigatti, Ana Beatriz Arêas da Luz Fontes

WHITFORD, V.; TITONE, D. Second-Language Experience Modulates Eye Movements During First- and Second-Language Sentence Reading: Evidence From a Gaze-Contingent Moving Window Paradigm. Journal of Experimental Psychology: Learning, Memory, and Cognition, v. 41, n. 4, 2015, pp. 1118-1129.

WONG, Y. K. Exploring the reading-writing relationship in young Chinese language learners' sentence writing. Reading and Writing, v. 31, 2018, pp. 945-964.

WORDSMYTH. The Premier Educational Dictionary-Thesaurus, 2018. Available at: < https://www.wordsmyth.net/>. Last access: 04 Aug. 2018.

Recebido em: 30/09/19

Aceito em: 03/11/19 